This is not the final version. The fully published version of Zanni M., Sharpe T., Lammers P., Arnold L., Pickard J. (2021) Towards a BIM-Based Decision Support System for Integrating Whole Life Cost Estimation into Design Development. In: Toledo Santos E., Scheer S. (eds) Proceedings of the 18th International Conference on Computing in Civil and Building Engineering. ICCCBE 2020. Lecture Notes in Civil Engineering, vol 98. Springer, Cham can be found at https://doi.org/10.1007/978-3-030-51295-8_16 


\title{
Towards a BIM-based Decision Support System for Integrating Whole Life Cost Estimation into Design Development
}

\author{
Mariangela Zanni ${ }^{1-2}$, Tim Sharpe ${ }^{1}$, Philipp Lammers ${ }^{2}$, Leo Arnold ${ }^{2}$, and James Pick- \\ $\operatorname{ard}^{2}$ \\ ${ }^{1}$ Strathclyde University, Department of Architecture, 16 Richmond Street, Glasgow G11XQ, \\ UK \\ ${ }^{2}$ Cartwright Pickard, 1 Canal Side Studios, 8-14 St Pancras Way, London NW1 0QG, UK
}

\begin{abstract}
A common barrier to achieving design intent is the absence of information about operational performance during key design stages. This results in uninformed decision-making, which has negative impacts on actual building performance and Whole Life Cost (WLC). It is proposed that Building Information Modelling (BIM) has the potential to assist design decisions at initial stages, if the model can utilize reliable and robust cost and performance data from buildings in use. This paper describes the findings of a research project that has investigated the integration of WLC estimation into BIM processes. Firstly, the research attempts to gather tacit expert knowledge by reverse-engineering unstructured processes to clarify information requirements for WLC assessment. The Integrated DEFinition (IDEF) 3 structured diagramming modelling technique was utilised for process mapping of information flows. As a result, value adding information, and the chain of interdependencies, were determined. The model has informed the development of a decision-support tool that automates prescriptive tasks and streamlines information exchange procedures. The research findings support the idea that BIM can assist the accurate estimation of WLC of an asset, from the early design stages, through the implementation of a structured information exchange. However, source data is fragmented and in propriety formats and further work is needed to standardise and gather performance data and BIM model structures.
\end{abstract}

Keywords: Building Information Modelling (BIM), Whole Life Cost (WLC), Process modelling, Integrated DEFinition (IDEF) language, Decision support.

\section{Introduction}

Successive UK construction industry reports have documented the fact that the sector has been suffering from low innovation and underperformance in terms of productivity and quality of the final product [1-3] and this has led to the UK Government's target of a 33\% reduction in WLC $[2,4]$. Research has shown that over a 30-year life span, the cost of operating the asset could be as much as four times the cost of designing and constructing the building, and that $80 \%$ of the operation, maintenance, and that 
replacement costs of a building can be influenced in the first $20 \%$ of the design process $[5,6]$. However, buildings rarely perform as expected [7,8]. This can impact on energy consumption, environmental quality and operational costs (OpEx) [5,9]. It has been argued that the most common barrier to achieving design intent is the absence of reliable predictive information during design and construction stages, leading to poor decision-making, which impacts on performance and WLC [10]. Furthermore, researchers have identified the lack of standard methods and format for calculating WLC as the main barrier for its implementation [11].

As a consequence, the need for a more holistic approach to building design is gaining momentum. BIM has proven its potential to facilitate the building development process during planning, designing, construction, and operation [12]. Nevertheless, to date, BIM has focused on the production of construction information and although the use of BIM for calculating CapEx has become commonplace, its potential to be used for estimating WLC is not yet developed. Several BIM software solutions have been developed with the aim of calculating WLC, but none of them offers a complete solution $[13,14]$.

This paper is based on a project that responds to the objectives of the UK Government's Transforming Infrastructure Performance (TIP) strategy [15] for adopting cost, schedule and performance benchmarks to support the selection, budgeting and design of projects through the development of disciplined definitions of required outcomes, ensuring they can be delivered in line with whole-life performance, cost and schedule targets. The project also addresses the objectives of the Construction Sector Deal (CSD) [4] by providing a common data framework and technology platform that support the design, delivery, and operation of higher performing buildings with lower carbon emissions and WLC. This can be achieved by harnessing the power of digital technology and data analytics [16]. The project focuses on the Build-to-Rent (BTR) housing sector $[13,17,18]$ as an area in which WLC can impact on commercial viability.

\section{Research strategy and methods}

This research follows the pragmatic paradigm throughout the research design, data collection, and analysis. Mixed methods were utilized [19], and rationalized through abductive reasoning [20] for the development and validation of a structured standardised process for more accurate and timely WLC estimation through BIM. Interviews, workshops, and the IDEF3 structured diagramming technique were utilized to identify valueadding tasks and coordinate them into a holistic process. Triangulation of qualitative and quantitative methods served to increase the validity and reliability of the data collected. The research process followed the Technology Readiness Level (TRL) framework [21]. Exploratory research served to satisfy the Discovery stage (Levels 1-2). Process mapping coordinated the components of the process into a structured framework and model (Levels 2-3). Computer programming and coding resulted in an automated, streamlined prototype tool that facilitated the standardised process (Levels 4-5). The next stages of development will be extrapolating lessons learnt into a large scale fully 
functioning software application that can be validated and marketed into a wide range of scenarios (Levels 6-9).

\subsection{Conceptual process modelling}

A conceptual model, in this study, is defined as a set of constructs, inferred by observable events, used to describe an event, object, or process. Thus, the identified propositions in a conceptual model are logical rather than epistemological. The chain of interdependencies was coordinated explicitly into a reverse-engineered systematic process that uses the IDEF3 structured diagramming modelling technique [22]. The IDEF3 method was selected due to its high descriptive power, which is considered appropriate for detailed processes that handle know-how knowledge. IDEF3 captures descriptions about sequences of activities, while also identifying critical decision points, or milestones, of the process from different perspectives [22,23]. The main assumption of the systemic model is that it represents an open system, hence its functions (i.e. tasks) need to be descriptive rather that prescriptive (see Fig. 1). Nevertheless, information flows and ranges of values (i.e. rules) were prescribed.

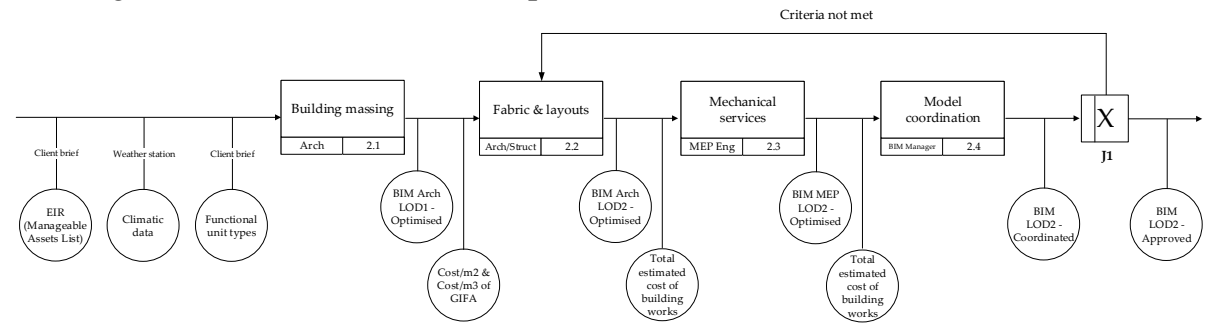

Fig. 1. IDEF3 decomposition of Stage 2 of design development

\subsection{The case study - Proof of concept}

The research implemented a case study approach to validate the efficacy of the developed concept. Case study approach is recommended when an in depth exploration of a situation is required, with emphasis on understanding processes as they occur in their context [24]. The selected building was a BTR scheme situated in Northwest London. The design team followed the IDEF3 modelled process to create BIM models in Autodesk Revit. Three levels of design development were implemented (see Fig. 2).
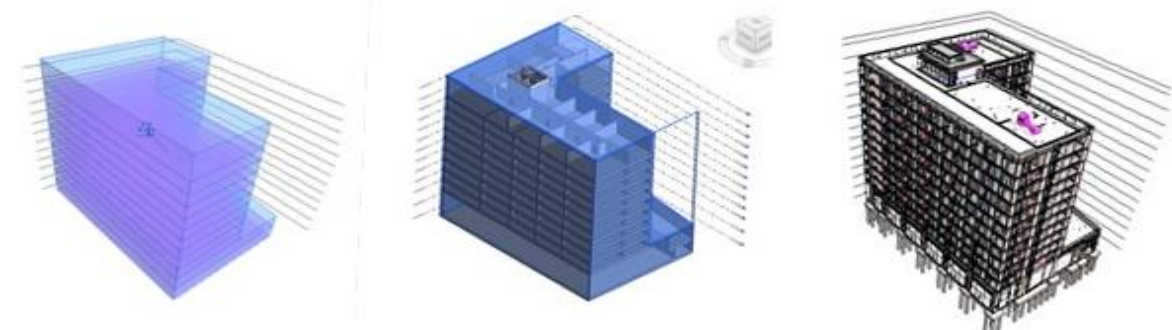

Fig. 2. Levels of model development (i.e. massing, functional units, and components) 


\section{Level 1 - Massing model}

The Proof of Concept model was developed in accordance with the IDEF3 definition using the Revit Conceptual Mass / Mass in Place tool. Mass Floors are activated automatically by defining Revit Levels in the BIM model. Roof level were excluded as this led to incorrect calculation of the GEA. Furthermore, internal voids and double spaces were not considered at this stage. The number of apartment types and auxiliary spaces were calculated using a Unit Mix Tool.The Massing model contained a single Uniclass 2015 code to determine the overall use of the building. In this case, Entity code Residential buildings (En_45_10) was selected. The Unit Mix Tool was aligned with more detailed SL (Spaces and Locations) codes for the different types of uses.

\section{Level 2 - Functional unit model}

In the Functional unit model, external walls, floors and roofs were added, applying the 'by face' option of Revit. Stair wells, lift and riser shafts were created using Shaft Openings with symbolic lines. Functional units were defined using the Rooms capability. Types of Functional unit include: Apartments, Circulation Spaces, Plant and other Auxiliary Spaces (Refuse and Bike Storage), Resident's Lounge, and Lobby. SL, EF, Ss, and Pr Uniclass2015 codes were used to specify Spaces and Locations, Elements and Functions, Systems, and Products respectively.

\section{Level 3 - Component model}

The Component model represented the highest level of granularity and detail. It is the level where each product was specified as close to the as-built as possible. At pre-construction stage, the performance specifications were predicted. These values could be substituted with actual performance by performing post-occupancy evaluation studies and updating the digital twin accordingly. SL, EF, Ss, and Pr Uniclass 2015 codes were used to specify Spaces and Locations, Elements and Functions, Systems, and Products respectively, as above. In addition to these, detailed specifications were selected for each product type using the naming of the NBS Product Data Templates (PDTs) [25].

\section{Discussion of research findings}

The research findings support the idea that BIM technologies can assist in the accurate estimation of WLC of an asset, from the early design stages, through the implementation of standardised information exchange procedures. Despite the potential, there remain many challenges to be faced before accomplishing fully automated, integrated, and interoperable data. This section discusses the challenges encountered for the standardisation and automation of the BIM-enabled WLC assessment process. It describes the principles that underlie BIM-enabled WLC estimation (section 3.1) along with the development of a small-scale prototype application that validates the analytical predictions and tests the feasibility of the system in a relevant environment (section 3.2). 


\subsection{Standardisation of the design process}

The main challenge in the attempt to standardise the early stages of the design process is that the problem is not sufficiently defined yet [26,27]. In addition to that, design problems are often idiosyncratic and difficult to understand [28]. Therefore, the process of design is highly iterative, and the experimentation and comparison of alternative solutions is necessary [29]. The IDEF3 model addresses this by incorporating design loops as part of the process, until certain conditions are satisfied. The tool has addressed this issue by reverse-engineering the process bottom-up by identifying key performance indicators in terms of functional performance. Standardisation of WLC information used the Uniclass2015 coding system [30] and data from several databases (i.e. RICS, BCIS, IFPI). For WLC assessment to become meaningful, material specifications, for example, $\mathrm{k}$-values, and recycled content should become explicit for the calculations to be accurate. Otherwise, the range of possible costs is so broad that the committing to a decision cannot be made with confidence. For example, for aluminium double-glazed windows, the following parameters were considered: (i) Thermal conductivity (U-values), (ii) Sound transfer (dBs), (iii) Fire rating, (iv) Strength, (v) Translucency, (vi) Security, and (v) Cleanability. Nevertheless, the level of information contained in BIM components is not comprehensive enough to enable the objects to be associated with WLC datasets. Key assumptions regarding several specifications (e.g. materials, properties) need to be made very early in the process.

\subsection{Sources of data - Data availability}

The accuracy of WLC substantially depends on the quality and availability of data from all phases of a building. It was found that historical databases contain several irregularities and ambiguities, and they don't offer neither the granularity nor the classification and clustering that is required to be used in an effective way. Datasets are not annotated consistently and the specifications and factors affecting WLC (such as intensity of use, environmental conditions) are not parametric. Moreover, annotations of datasets are not aligned with those in BIM entities. Therefore, direct alignment to model objects was not possible. Furthermore, accessibility to WLC information presented the biggest challenge, as it is either stored in static documents, or it is proprietary. Data from manufacturers, suppliers, and specialist subcontractors is also difficult to obtain due to concerns regarding liability and commercial competitiveness. Consequently the level of detail of existing readily available datasets is not appropriate to be used in conjunction with BIM. It was inferred that collection of in-use data, which is fit-for-purpose, can be a viable solution however at present there is a paucity of longitudinal studies that collect data on the performance and reliability of systems. BIM-enabled WLC integration is also hindered by the fact that existing databases are static, are not open source, and contain insufficient and unproperly structured information. This is mainly due to failures of the industry to collect WLC information but also due to lack of understanding of what information is required. For a BIM-enabled WLC estimation process, data need to be structured and formatted in a way that communicates with existing systems and calculations tools to become useful. 


\subsection{System architecture - Modules and connections}

The prototype software tool performs the following functions: (i) Extraction of area measurements, functional units, and component quantities out of BIM models of varying levels of design development (massing, concept, developed, and technical design in CSV and JSON file formats); (ii) Uses WLC benchmarks from BCIS and BLP to create data structures that combine NRM/BCIS codes with Uniclass2015 for varying levels of granularity; (iii) Performs WLC calculations using quantities extracted from the BIM model (through a Revit plugin combined with Excel spreadsheets); and (iv) Exports the result of calculations to Microsoft PowerBI for the creation of dashboards. It consists of the following modules, illustrated in Fig. 3.

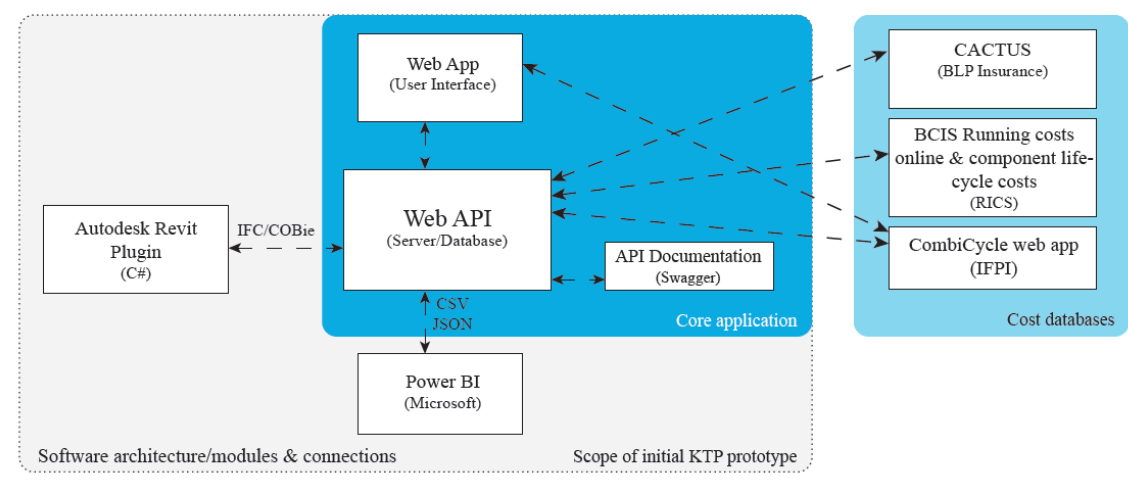

Fig. 3. Software architecture and connections

\section{Autodesk Revit plugin - Quantity take-off}

As described in section 3, quantity take-off for the Massing and Functional units' models (Levels 1 and 2) is the square meterage of useable areas. These quantities were then multiplied using benchmarks acquired by the RICS' BCIS Running Cost Online subscription service. It needs to be stressed that, for the quantity extraction process to work, the BIM model needs to be built using a prescriptive method both for the geometric and non-geometric information. Apart from the example of the Roof and Rooms discussed above, the Uniclass2015 classifications of the components need to be specified in advanced as part of the Employer's Information Requirements (EIR). Several workarounds and bespoke solutions had to be developed for extracting the quantities of the architectural elements due to limitations of Revit. For example, Revit calculates the area of a window as a box, while the WLC calculations require the area of the opening. Inaccuracies in quantity extraction have also been noticed for the structural elements where there were intersections between columns and slabs. The solution that was implemented in this occasion was to use the "Join" tool so that the tool considers only one of the elements. This is a limitation of the process as well, since at early design stages it is not efficient to make these adjustments as they are time consuming. Another challenge was that the MEP BIM model was not comprehensive enough to be used as a 
system. For that reason, the proof of concept utilised IFPI's parabolic curves for estimating cost, carbon, and energy consumption of extract ventilation systems for 50 years based on the ratio of wet areas to total GIA of the building, which differs between typologies. It is suggested that future work should incorporate the Industry Foundation Classes (IFC) data structure to overcome the inherent limitations of Revit, as it offers dedicated information containers for model components and information. Work is also needed to develop structured processes for model construction by designers.

\section{Web API application - Server (database and user interface)}

The Web API application contains the re-structured datasets of cost and carbon information. This data was pre-processed; adjusted for London and to represent totals for a 50-year calculation period. Some datasets were also annualised to be used for creating more detailed breakdown of cash flows. Discount factors were applied separately in PowerBI. All components were classified using Uniclass 2015 codes and were linked to the BIM model through the identification of identical classifications. For the Proof of Concept, all component and database classifications were specified explicitly in advance. For future development, the software should be adjusted to find the closest match and present the alternative cost and carbon information available in the database. The database follows an object-oriented structure where components were defined by Pr (Product) codes and functional units (i.e. Revit Rooms) by SL (Space and Location) codes. Relationships between Components and functional units are also built in. All entities have a list of properties attached to them and they are also defined by a Revision that is associated to stage of design development and a user of the system. The user interface of the web application is accessible via a browser. This solution was preferred to a desktop application as it offers better accessibility. Additionally, the web API is supplemented by API documentation developed using Swagger. This contains instructions on how to integrate the web application with external applications, offering the option to create connections to a multi-platform structure.

\section{$4 \quad$ Conclusions and future work}

To date, key decisions (relating to WLC) are often based on judgement rather than data, which leads to an increase in the level of risk and uncertainty. There is no comprehensive tool to assist designers commit to decisions in a timely manner and with confidence. The scope of the project was to integrate cost and carbon information related to maintenance and operation of a completed building throughout design delivery, from the early stages, as an integral part of the natural design development process. The Proof of Concept case study focused on the BTR housing sector, for whom accurate WLC can influence long term financial viability. Its main objectives were: (i) to investigate the emerging landscape of BTR housing, establish the need and opportunity for WLC assessment, and explore the capabilities of BIM to facilitate this purpose from the early design stages, and (ii) to develop and verify a structured standardised process for more accurate WLC estimation through BIM and assess the benefits of this innovative 
approach. As a result, the project proved the potential of BIM to accelerate the process and reduce the tedious and repetitive tasks that are currently used to assess WLC. It demonstrated how BIM has the potential to facilitate more accurate prediction of WLC more efficiently in terms of time and effort involved so as to achieve quality assurance. As such, WLC can consider all significant and relevant initial and future costs and benefits of an asset, throughout its life cycle, while fulfilling the performance requirements. This holistic approach can result in high-performance buildings that are cost-effective, thus sustainable.

\subsection{Challenges and limitations}

The project identified several issues that are barriers to BIM-enabled WLC estimation. These can be summarised as follows:

- Lack of a common standardised framework and method that provides appropriate definitions of information requirements and is aligned with BIM standards and protocols for delivering design information which is fit-for-purpose for WLC estimation.

- Alignment of modelled elements, annotations, and properties to information contained in cost databases. Models are constructed ad hoc at early design stages in order to produce quick 3D information and restructuring these later is time-consuming and generates inaccuracies.

- Availability, accuracy, reliability of cost and performance data as well as accessibility and structure of available data. WLC and performance data is not routinely collected from existing buildings. Consequently, data that exists is small-scale, fragmented, held in propriety formats and is not open source.

- Proprietary file formats of BIM authoring software are restricting the extraction of information from the models. Implementation of open standards has the potential to facilitate transparency and information exchange procedures.

\subsection{Future work}

Based on the findings above, the next stage of the project will focus on:

- Facilitating standardised procedures for the collection, structuring, and analysis of WLC data in order to comply with the granularity of the Uniclass2015 classifications. This approach will allow benchmarking at varying levels of detail for both areas and components, based on UK standards. Whilst government is mandating use of BIM, this is not extended to performance data, and this is vital to the accuracy and robustness of modelling tools.

- Development of structured and standardised approaches for model construction at design stages to enable increased granularity of data at later design stages. All levels of geometric development need to be populated with appropriate information that is useful for WLC assessment so as to enable association of objects to WLC datasets.

- Developing a web-based Software-as-a-service (SaaS) application that links WLC calculations to BIM authoring tools for extracting quantities natively and performing calculations "on-the-fly" so as to streamline integrated WLC assessment. 
- Re-structuring datasets of existing historical databases that contain WLC information by clustering and classification. These will be used to populate and maintain a dynamic WLC database and use it to identify patterns, dependent variables, and predict future performance.

- Testing the effectiveness of visualisation methods that assist the interpretation of WLC estimations and determining the ones that have the most significant effect on improving the final design product/solution.

\section{References}

1. Cabinet Office. Government Construction Strategy, May 2011; GOV.UK (2011).

2. Government, H. M. Construction 2025: strategy https://www.gov.uk/government/publications/construction-2025-strategy.

3. Farmer, M. The Farmer Review of the UK Construction Labour Model (2016).

4. HM Government. Construction Sector Deal (2018).

5. BSI. ISO 15686-5:2017 - Buildings and Constructed Assets -- Service Life Planning -Part 5: Life-Cycle Costing (2017).

6. White, G.; Boyne, P. Facilities Management. In BIM and Quantity Surveying; Routledge (2016).

7. BSRIA. BG 64/2016 - Building Performance Evaluation in Domestic Buildings - a BSRIA Guide (2016).

8. Sharpe, T.; McGill, G.; Gupta, R.; Gregg, M.; Mawditt, I. Characteristics and Performance of MVHR Systems - A Meta Study of MVHR Systems Used in the Innovate UK Building Performance Evaluation Programme (2016).

9. RICS. RICS Professional Guidance: Life Cycle Costing, 1st ed.; RICS (2016).

10. Zanni, M. A.; Soetanto, R.; Ruikar, K. Towards a BIM-Enabled Sustainable Building Design Process: Roles, Responsibilities, and Requirements. Archit. Eng. Des. Manag. 13 (2), 101-129 (2017). https://doi.org/10.1080/17452007.2016.1213153.

11. Opoku, A. The Application of Whole Life Costing in the UK Construction Industry: Benefits and Barriers. Int. J. Archit. Eng. Constr. 2 (1), 35-42 (2013). https://doi.org/10.7492/IJAEC.2013.004.

12. Eastman, C. C.; Teicholz, P.; Sacks, R.; Liston, K. BIM Handbook: A Guide to Building Information Modeling for Owners, Managers, Designers, Engineers and Contractors, 2nd ed.; John Wiley \& Sons (2011).

13. Zanni, M.; Sharpe, T.; Lammers, P.; Arnold, L.; Pickard, J. Developing a Methodology for Integration of Whole Life Costs into BIM Processes to Assist Design Decision $\begin{array}{llllll}\text { Making. } & \text { Buildings } & 9 & \text { (5), } & 114 & \text { (2019). }\end{array}$ https://doi.org/https://doi.org/10.3390/buildings9050114.

14. Kehily, D.; Underwood, J. Embedding Life Cycle Costing in 5D BIM. J. Inf. Technol. Constr. 22 (August 2016), 145-167 (2017).

15. HM Government. Transforming Infrastructure Performance (2017).

16. Centre for Digital Built Britain. Gemini Principles (2018).

17. Urban Land Institute (ULI). Build to Rent Best Practice Guide: 2nd Edition, 2nd ed.; ULI UK Residential Council (2016). 
18. Mayor of London. Homes for Londoners: Affordable Housing and Viability Supplementary Planning Guidance 2017 (2017).

19. Creswell, J. W. Research Design: Qualitative, Quantitative, and Mixed Methods Approaches; Sage (2009).

20. Dubois, A.; Gadde, L.-E. Systematic Combining: An Abductive Approach to Case Research. J. Bus. Res. 55 (7), 553-560 (2002). https://doi.org/10.1016/S01482963(00)00195-8.

21. Nuclear Decommissioning Authority. Guidance on Technology Readiness Levels (2014).

22. Mayer, R. J.; Menzel, C. P.; Painter, M. K.; Dewitte, P. S.; Blinn, T.; Perakath, B. Information Integration for Concurrent Engineering (IICE) - IDEF3 Process Description Capture Method Report; DTIC Document (1995).

23. Mayer, R. J. IDEF Family of Methods for Concurrent Engineering and Business ReEngineering Applications; College Station, Texas, USA (1992).

24. Yin, R. Case Study Research: Design and Methods; SAGE Publications (2013).

25. Jones, L. Product Data Templates For Manufacturers https://toolkit.thenbs.com/articles/pdts.

26. Sebastian, R. Managing Collaborative Design; Eburon Uitgeverij BV (2007).

27. Dorst, K.; Cross, N. Creativity in the Design Process: Co-Evolution of ProblemSolution. Des. Stud. 22 (5), 425-437 (2001).

28. Laseau, P. Graphic Thinking for Architects \& Designers; John Wiley \& Sons (2001).

29. Goldschmidt, G. Linkography: Unfolding the Design Process; The MIT Press: Cambridge, Massachusetts ; London (2014). https://doi.org/10.1016/j.destud.2015.10.003.

30. NBS. Uniclass 2015 - a universal classification system for the construction industry | NBS https://www.thenbs.com/news/uniclass-2015-a-universal-classification-systemfor-the-construction-industry, last accessed 2018/02/05. 